\title{
A Model for Content Representation of Multimedia Information
}

\author{
Artur Caetano \\ Instituto Superior Técnico, Technical University of Lisbon, \\ Lisbon, Portugal \\ amc@asterix.ist.utl.pt \\ Nuno Guimarães \\ Faculdade de Ciências de Lisboa, University of Lisbon, \\ Lisbon, Portugal \\ nmg@di.fc.ul.pt
}

\begin{abstract}
This paper presents a generic model to describe the content and structure of video and image data. A video model is used to create a set of hierarchic groups that describe the structure of a video, allowing semantic concepts, such as scenes, to be represented. The description of the video content includes temporal features extracted from the video shots and spatial features of the key-frames. We describe the method of creating a complete description for video material at three different levels of abstraction: group, shot and key-frame. We also describe a heuristic process to extract the key-frames from a video shot.
\end{abstract}

\section{Introduction}

Due to the advance of computer and communication technologies, large volumes of multimedia information can be easily obtained. Despite this availability, some content-based information and retrieval systems still rely on text-based mechanisms to access video and image data. Moreover, considering this material as an unstructured and opaque data type, makes difficult its manipulation, browsing and retrieval. To handle effectively this type of information, we require description models that are aware of the structure and content of the multimedia data together with effective algorithms for feature extraction. This paper describes a model for organizing the features of multimedia data, aiming at its description and indexing. This model is being used in a content-based information and retrieval system for video and image data, which is currently under development.

The primary step in structuring video information consists on finding its basic units, or shots, through temporal segmentation. A shot is defined as a sequence of one or more frames that were contiguously recorded and that represent a continuous action in space and time. Therefore, each shot is separated by some type of transition, either abrupt or gradual. To detect these transitions, several approaches are described in the literature, either using the properties of the video frames $[14,21,26]$, or by analyzing directly the compressed video data $[22,25]$

However, using the video shots as the only means to organize the video information is clearly not enough to express its semantic properties. To organize the video at a semantic level, two different approaches exist: general-purpose and model based. Aoki et al and Yeung et al presented general-purpose methods where clusters of shots are created according to visual and temporal features. Then, a scene transition graph is created using the cluster and "cutting edge" information [2]. In [23], instead of generating the scene graph, the clusters are organized into groups of similar patterns, named acts.

The model based organization methods, require a specific model for the video domain to be first created. This model contains a priori knowledge that specifies the conditions that allow the video shots to be organized into a semantic structure. For example, Zhang et al [24] uses this approach to create a hierarchy of semantic units for newscasts, based on the detection of anchorperson shots. We have previously presented a modeling language and editing environment that allows defining the conditions to group together multiple shots [4]. This hierarchy of groups is able to represent semantic concepts, such as scenes and other types of logic groups. This video hierarchy also facilitates the video browsing and searching processes.

With an adequate video model, i.e., a model that accurately describes the video organization, we are able to browse and retrieve a video according to its structural properties. To extend the retrieval process to the video content, we further need to extract a set of low-level features from the video shots, which can be related to the temporal events of the groups, or to the spatial features of its frames. However, effective indexing and retrieval methods are still a research problem when feature sets of large dimensions are considered. One of the most common indexing structures, the $\mathrm{R}$-tree and its variants, such as the $\mathrm{R}^{*}$-tree, prove not to be adequate for indexing high-dimensional data sets [2]. Therefore, other structures, such as the VAM Split k-d tree [20] and the X-tree [2] have been proposed. 
Although most several video and image indexing and retrieval systems make use of primitive or lowlevel features $[6,7,9,14,15,27]$, there have been other approaches which use high-level concepts. Rowe et al presented a system that relies on textual indices to describe video information [16]. Text-based descriptions can be detailed and rich, but its generation is difficult to automate and they can be ambiguous. Given the problems related to natural language descriptions, Davis proposed an iconic language to annotate and index video, which is able to describe complex and semantically rich concepts [11]. Though the obtained results are encouraging, the system must be considered in a specific domain of application to be useful, since the description process is not automated and can be complex.

In this paper, we propose a multimedia representation model to describe image and video data, allowing multiple temporal and spatial features to be specified. This structure allows the data to be retrieved according to its content and structure. The model also provides a platform for video browsing and navigation. This paper is organized as follows: Section 2 depicts the model for multimedia information. Section 3 reviews the process of video representation, including temporal segmentation, video abstraction. The indexing process is described in Section 4. Finally, Section 5 contains the conclusions and an overview of our future work.

\section{Multimedia Content Representation}

In the domain of image data, some description structures have already been proposed [17]. We have extended the underlying concepts of those models so that other data - especially video data - may also be considered. In this approach, a multimedia object contains the representations required to describe a basic unit of information, such as an image, a video shot, an audio clip or even a structured unit, such as a video scene. After indexing, the objects can be retrieved according to their content and structure.

A multimedia object is defined as $O=\left(O_{\text {id }}, O_{\text {type, }} F\right)$, where $O_{\text {id }}$ is a unique identifier for the object. $O_{\text {type }}$ depicts the type of data the object is describing, and can be one of shot, group, key-frame or image. $F$ is the generic feature set associated with this object, used to represent features such as color, texture, shape, etc. Each feature is composed of one or more representations. For example, the color feature may have several representations, such as the color histogram and the color moments. Each representation is a $n$-dimensional vector, where $n$ depends on the specific representation size. These representation vectors are indexed in a multidimensional structure using the object identifier $O_{\text {id }}$ as primary key. We are using the $\mathrm{X}$-tree as indexing structure, since it is capable of efficiently managing large volumes of high-dimensional data [3].

Besides the representation vectors, meta-information, which is represented as a collection of textual features, is also be included in the feature set to enable higher-level descriptions of the data. It currently contains a set of keywords that describe the content of the object, a set of URLs to other relevant material and other information, such as the title of the object, author and date. This information is being indexed in a relational database. However, other structures can be more adequate to index and retrieve text, like objectoriented databases or special purpose structures, such as B-trees.

\subsection{Query representation}

Querying is the process of retrieving the $k$ most similar objects to a set of feature vectors that designate the query's content. We represent a query as an extended multimedia object $Q$, defined as $Q=\left(O_{\mathrm{Q}}, M\right)$, where $O_{\mathrm{Q}}$ contains the features that describe the query, and $M$ is the set of similarity functions required to compute the overall similarity value. Each representation in the object is associated with one metric, enabling different similarity functions to be used within the same query. For example, if a query consists of vectors describing the color histogram and the CCD texture features, we can use the histogram intersection function to compute the similarity between histograms, while using the Mahalanobis distance function to compare the texture vectors. Additionally, each feature and representation in $O_{\mathrm{Q}}$ may have associated a weight, i.e.:

$$
O_{Q}=\left\{\left(f_{1}, W f_{1}\right), \ldots,\left(f_{n}, W f_{n}\right)\right\} \text { with } f_{i}=\left\{\left(r_{i, 1}, W r_{i, 1}\right), \ldots,\left(r_{i, m}, W r_{i, m}\right)\right\}
$$

where $W f_{1}$ is the weight of the feature $f_{\mathrm{j}}$ and $W r_{\mathrm{j}, \mathrm{k}}$ is the weight of the $k$-th representation of the $j$-th feature. All the weights have a value in the interval $[0,1]$. The similarity between a query $Q$ and another multimedia object $O$, is given by:

$$
\operatorname{Sim}(Q, O)=\sum_{i=1}^{n} \sum_{j=1}^{m} W f_{i} \times W r_{i, j} \times m_{i, j}\left(r_{i, j}, r_{i, j}^{\prime}\right)
$$

where $m_{\mathrm{i}, \mathrm{j}} \in M$ is the function that computes the normalized similarity between representations $r_{\mathrm{i}, \mathrm{j}}$ and $r_{\mathrm{i}, \mathrm{j}}$ in the interval [0,1], $r_{\mathrm{i}, \mathrm{j}}$ is a representation vector of $Q$ and $r_{\mathrm{i}, \mathrm{j}}$ is the corresponding representation on the object $O$.

Besides simple queries, we allow several query specification objects to be combined using three logic operations (and, or, not), thus creating a composite query. The overall similarity before normalization between a composite query $C Q$ and a multimedia object $O$ is given by the membership value in the fuzzy sets represented by the query objects that comprise $C Q$, where $Q_{\mathrm{i}}$ is a simple query and $o p_{\mathrm{i}}$ is a logical operator: 


$$
\begin{aligned}
& \text { OverallSim }(C Q, O)=\operatorname{CompSim}\left(o p_{1}, Q_{1}, Q_{2}, O\right)+\ldots+\operatorname{CompSim}\left(o p_{m}, Q_{n-1}, Q_{n}, O\right)
\end{aligned}
$$

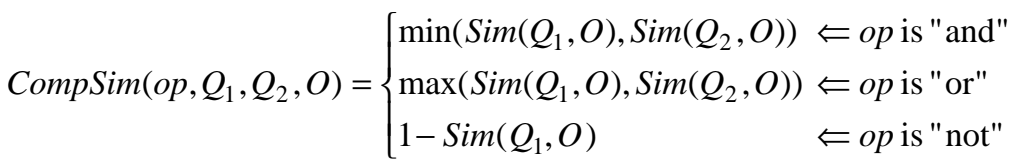

\section{$3 \quad$ Video Representation}

We consider three levels of abstraction to describe the structure and the content of video material. The group level describes the properties of semantic units of video, such as scenes, and consists of a set of adjacent shots or other groups. The collection of groups can be viewed as a tree that represents the semantic structure of the video material. The shot level describes the video units using its temporal features or other meta-data. Finally, the frame level contains the description of the visual features related to a single key-frame of a shot. The video description process can be summarized in the following steps:

- Find the transitions between the shots of video data by temporal segmentation.

- $\quad$ Extract the temporal features associated with each shot, creating the shot level descriptions.

- $\quad$ Extract a collection of relevant frames, or key-frames, from each shot.

- $\quad$ Extract the visual features from every key-frame, creating the frame level descriptions.

- Create a tree of video groups using the shots and other groups as nodes according to the grouping conditions on the video model. This tree will describe the video structure.

- Extract the properties of each video group, creating the group level descriptions.

- $\quad$ Create and index the multimedia objects for the descriptions at frame, shot and group levels.

We have proposed a video modeling language to specify the video structure and a computer-assisted environment that allows video models to be visually specified, enabling complex grouping conditions to be defined [4]. The grouping conditions of the video shots are based on the primitive properties of the frames and shots (v. Figure 1). Besides defining the structure of the video, a group also contains meta-information concerning its description and the description of its sub-nodes (shots or other groups).
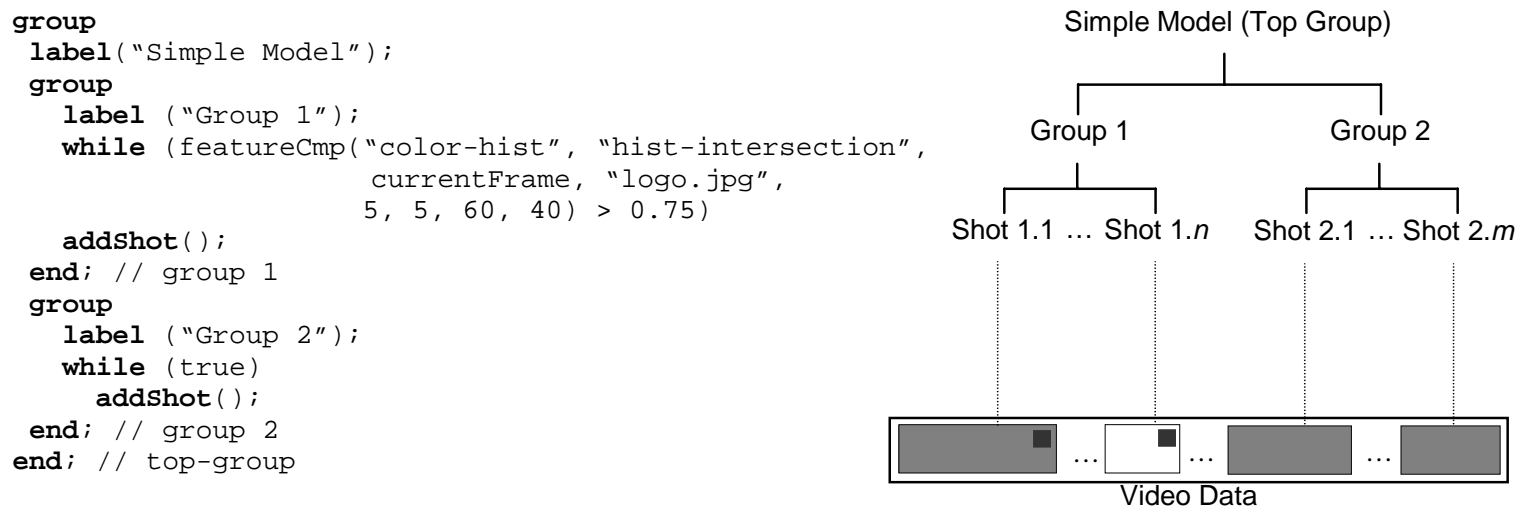

Figure 1: Example of a video model and the corresponding hierarchic structure. Three groups are specified: the "top group", representing the complete video structure; "group 1", containing all the adjacent shots matching a specific visual property; and "group 2", containing the remaining shots after the grouping condition becomes invalid. The main grouping condition specifies that while the chromatic histogram of the upper-left region of the first frame of the shot has a 0.75 similarity to some external image, the shot is to be included in the group (line 5). The external image can represent a logo that identifies a specific program, such as a caption in a newscast.

The structure description is not enough to effectively index the video data, since its content is not yet being considered. The content description will include the temporal events of the shots and the spatial information from a collection of key-frames. After the content and structure of the video data are obtained, we can map these descriptions into a collection of multimedia objects. We require three classes of multimedia objects, one for each level of representation: group, shot or frame. These objects only differ in the type of features and representations each contains. In the following sections, we will describe how to obtain descriptions for each of those objects. 


\section{Temporal Segmentation}

To find the transitions in a video sequence, and thus obtain the video shots, we use the net comparison algorithm, which detects transitions by comparing the color histogram in several regions of adjacent frames [21]. When the video is in MPEG format, we can use the motion vectors and the DCT coefficients to perform the segmentation without decompressing the video data. With this approach, it is possible to achieve fast processing and good segmentation accuracy $[1,22,25]$.

A multimedia object is created to hold the description of each shot. This description comprises metainformation, such as the duration of the shot, the number of key-frames and textual representations, and a set of primitive temporal and spatial features.

\subsection{Temporal Features of a Shot}

We need to extract the temporal events and properties of a shot to make possible the retrieval based on these features. The temporal features we are considering are:

- Camera operations: panning, zooming and tilting. This feature is obtained through the analysis of the motion vectors between consecutive frames.

- Variation of the dominant colors: the mean and variance of the dominant colors of each frame in the video shot (v. section 6).

- Dominant colors: the 16 dominant colors of all the frames in the video shot.

- Variation of the color moments: the mean and variance values of the color moments (v. section 6).

- Shot classification.

We have defined a set of pre-defined categories to classify the video shots. Although the categories we are considering are quite generic, this feature proves to be useful since it is able to discriminate the shot type and can be automatically extracted. The classification process relies on the analysis of the temporal and spatial features of a shot, and on the structure of the video model. Currently, we consider the following categories:

- Light conditions: dark, normal, light. This classification is obtained by analyzing the temporal color features of the shot.

- Movement: no movement, slow, medium, fast. Feature derived from the variance of the color features, motion vectors and number of potential key-frames from the shot.

- Location: outdoors, indoors. On indoor shots, the pixel differences between frames tend to appear in well-defined areas, contrasting with outdoor shots. Moreover, the motion vectors in indoor shots are not abrupt and follow well-defined paths. In the case of newscasts (and other programs), some a priori knowledge can be included in the video model enabling the automatic classification of a shot as indoors due to the presence of an anchorperson or specific logo

- Type: newscast, commercial, other. A commercial is usually characterized by a large number of keyframes and short duration, in opposition to a newscast. The commercials during the breaks of a program can be easily classified as such if we use a video model that identifies the start and end sequences of the program.

\section{Shot Abstraction}

To obtain the visual features regarding the content of a shot, it is not required or feasible to index all its frames, due to the redundancy and to the computational cost associated with this procedure. To overcome this problem, we abstract the video shot, i.e., reduce it to a set of representative key-frames, which should be able to capture the shot's context [5]. To do so, we have defined a method to automate the shot abstraction process using its temporal and visual features. In theory, the set of key-frames would contain all the frames comprising events, actions and objects of interest within the shot.

Each relevant frame is assigned a score depending on its ability to summarize the shot's content. The factors that contribute to the score of a frame are:

- The first and last frames of the shot are always key-frames.

- The frame is able to summarize a camera operation. The first and last frames of the operation are relevant, as well as one or more intermediate frames, depending on the duration of the operation.

- The frame contains sudden hue or brightness variations. These events are usually of importance since they may indicate lighting variations and object movements. To obtain these key-frames, we compare the variation of the color features between consecutive frames. 
- The color properties and the layout properties of the frame are not contained in the average values of the shot.

- The frame shares a common object or background. This feature is obtained by analyzing the color variance in specific regions of the frames.

- The frame has a high-degree of motion. This can be obtained through the analysis of the image's motion vectors.

To filter the selected frames, we decrease the score of each potential key-frame that describes the same event. Then, the set of key-frames with the highest score is selected. The number of frames in the set also depends on an adjustable "density" value. In the next section, we will describe the process of indexing a keyframe.

\section{Key-Frame Representation}

A key-frame is described through several automatically extracted primitive features. It is important to note that the key-frame representation procedure also applies to images not from a video source. In this section, we will briefly describe these features.

\subsection{Color}

Color has a very important role in image retrieval systems since it holds a large discriminating power. To represent color, we use the Munsell hue-saturation-value color space ${ }^{1}$, since it is closest to the human perception [12]. We are considering the following representations for the color feature:

- Dominant colors: The 16 dominant colors of the image, obtained by selecting the histogram bins with the higher pixel count.

- Color histogram: A 128 bin normalized color histogram. The distance $D$ between two histograms $P$ and $Q$, each composed of $N$ bins, is calculated using equation 1 . The elements of the matrix containing $a_{\mathrm{ij}}$ represent the proximity coefficients between the colors of bins $i$ and $j$, and are determined from human visual perception studies which measure the human ability to discriminate colors [10]. Experimentally, we have verified that this metric generally performs better than the histogram intersection function [8].

$D^{2}(P, Q)=\sum_{i}^{N} \sum_{j}^{N} a_{i j}\left(p_{i}-q_{i}\right)\left(p_{j}-q_{j}\right)$

- Color moments: We use the first three order moments to represent the distribution of each color component [18]. The first moment defines the average intensity (v. eq 6); the second defines the variance (eq. 7) and the third the skewness (eq. 8). $v_{\mathrm{k}, \mathrm{i}}$ is the $k$-th moment for the $i$-th color component; $p_{i, j}$ represents the value of the $i$-th color component for the $j$-th image pixel; $N$ is the total number of pixels in the image.

$$
\begin{aligned}
& v_{1, i}=\frac{1}{N} \sum_{j=1}^{N} p_{i, j} \\
& v_{2, i}=\frac{1}{N-1} \sum_{j=1}^{N}\left[p_{i, j}-v_{1, i}\right]^{2} \\
& v_{3, i}=\frac{1}{N} \sum_{j=1}^{N}\left[\frac{p_{i, j}-v_{1, i}}{\sqrt{v_{2, i}}}\right]^{3}
\end{aligned}
$$

\subsection{Texture}

Texture representation algorithms can either be statistic or transform based, existing several alternatives on each category, such as the Wold decomposition [15] and the shift-invariant eigenvector [13]. Currently we are only using the statistic-based Contrast-Coarseness-Directionality Tamura features [19].

\subsection{Edges}

We are applying a Söbel filter and a Gaussian noise-reduction algorithm to obtain the edge map for each image.

\footnotetext{
${ }^{1}$ Since the V component is the most sensible to lighting and brightness variations, we have set its weight to 0.20 after some experiments. The $\mathrm{H}$ and $\mathrm{S}$ components have a weight of 0.40 .
} 
We can then correlate the edge maps of different images, and obtain a similarity measure. However, the obtained results are not encouraging, since this metric is not invariant to the image orientation and size, limiting its domain of application.

\title{
6.4 Layout
}

The color and texture features are effective to describe a complete image. However, queries that make use of spatial locations are not possible, since the layout information is not considered when extracting these features. To overcome this limitation, we divide the image into nine rectangular regions and extract the color and texture features from each region [9].

\section{$7 \quad$ Multimedia Object Indexing}

As shown in the previous sections, the video data is described by several structures at group, shot and keyframe level. These descriptions specify the structure and content of the data through meta-information and primitive properties.

To index properly these descriptions, each object requires a unique identifier, $O_{\text {id }}$ for later retrieval. The identifier relies on the absolute "path" of the object in the video structure to guarantee its uniqueness. For the example in Figure 2, the path in solid black is identified by top-group/group $\mathrm{n} / q$, where top-group is the URL of the video data. A shot is identified by its relative position in the group, i.e., top-group/group $/ i$, where $i$ is the shot number. A key-frame is identified by top-group/group $/ i / j$, being $j$ the key-frame number. To describe single images, the identifier is the URL of the image. This hierarchy proves to be useful during the browsing of video data, since it is possible to navigate on the video and shot structure. It is also possible to browse the video using its key-frames as they summarize the contents of a shot.

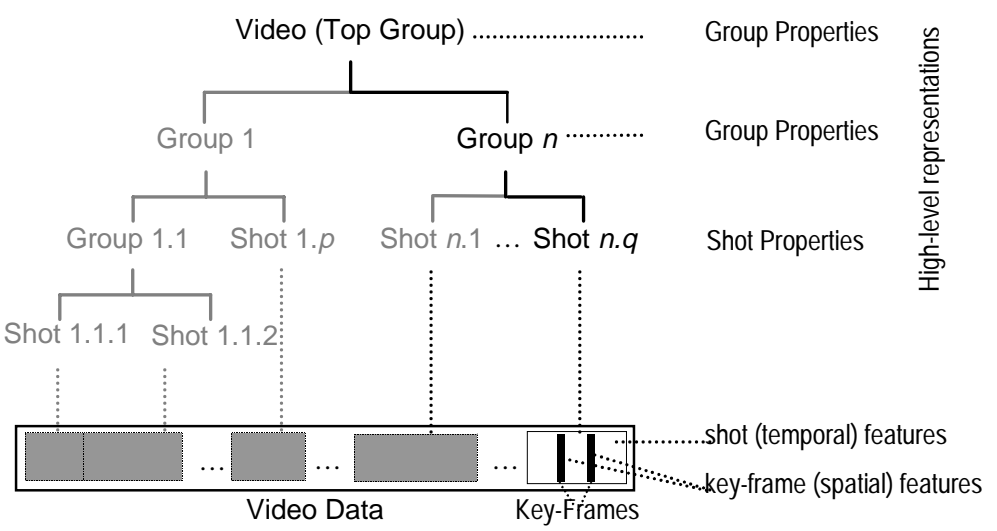

\author{
[Video] \\ group meta-data \\ [Group $n$ ] \\ group meta-data \\ [Shot $n . q$ ] \\ shot meta-data \\ shot temporal features \\ [Key-frame n.q.1] \\ frame meta-data \\ frame spatial features \\ [Key-frame n.q.2] \\ frame meta-data \\ frame spatial features
}

Figure 2: The structure of the video is represented by several group descriptions, while a set of shot and key-frame descriptions provide the content descriptions.

\section{Conclusions and Future Work}

The multimedia object model here described is designed to support multiple levels and types of features. These features describe primitive properties represented by multi-dimensional vectors, or generic meta-information. In either case, a similarity function is required to compare features of the same type.

In this work, the model was instantiated to video data, and, consequently, to single images. We are able not only to specify queries regarding the content of the video using the primitive features and meta-information available in the descriptions, but also its structure at three different levels of detail: group, shot and key-frame. Composite queries that use simultaneously content and structure descriptions are also possible.

To derive automatically the structure information, we are relying on a video model that uses a priori knowledge of the video material, and creates semantic groups of video data according to its primitive properties, thus exploiting its hierarchical nature. The content of the video is described using meta-information and low-level temporal information, automatically extracted from the video shots, groups and video model. Each shot is isolated using a color histogram-based metric, or, if the video is in MPEG format, the temporal segmentation is performed without decompressing the video data. A description of the visual content of the video data is obtained by indexing the most relevant frames from each shot. These frames are selected by a heuristic abstraction process that uses the temporal features of the shot and the spatial properties of its frames. The methodology used to index a video frame can also be applied seamlessly to isolated images from non-video sources.

The overall system architecture is depicted in Figure 3, showing the flow of information between the several processing stages. 
We are currently researching on the specification of complex query, i.e., queries comprising data from multiple levels of description, particularly describing temporal events. We plan to include shortly other feature extraction algorithms for video and image data, namely object and object movement detection, shape classification and audio analysis to help the classification of the temporal events.

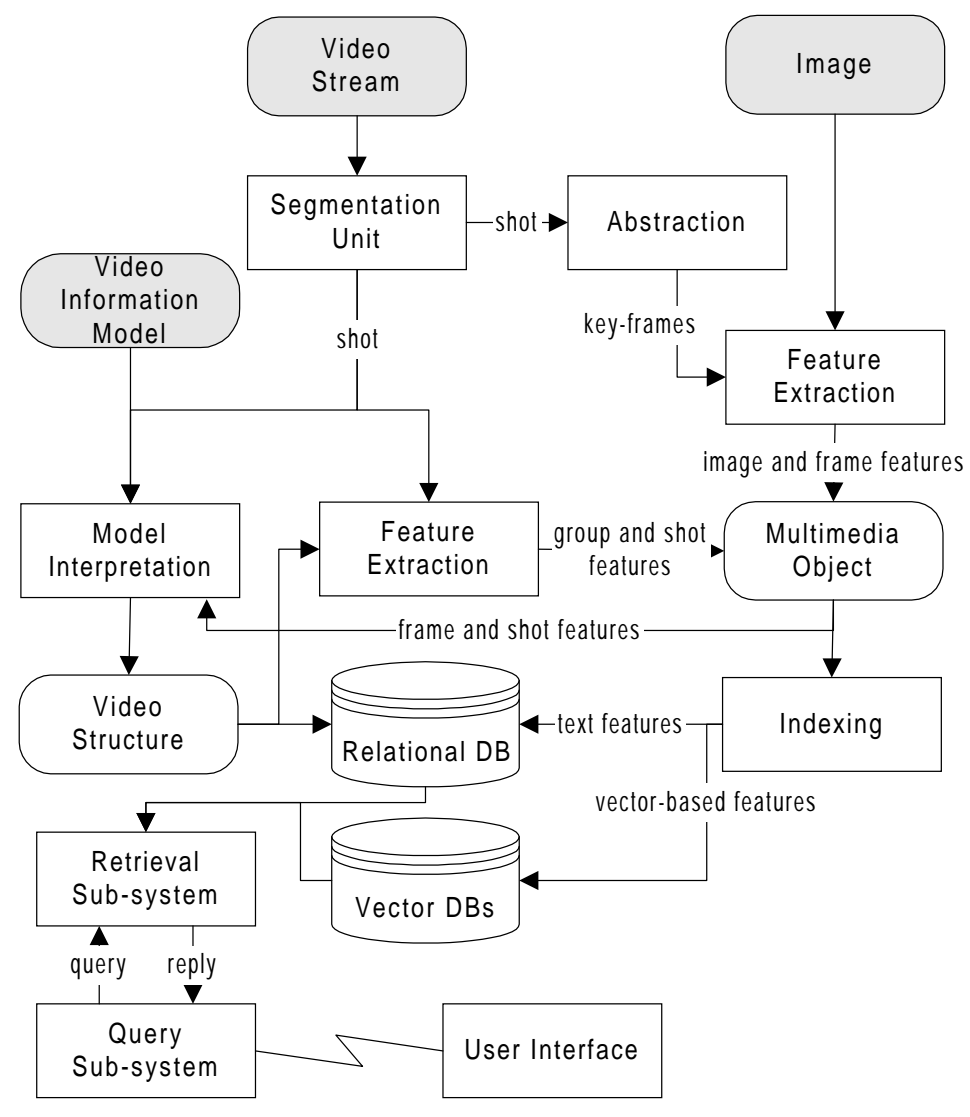

Figure 3: System architecture.

\section{References}

1. Akutsu A et al. Video indexing using motion vectors. In IS\&T/SPIE Visual Communication and Image Processing, 1992

2. Aoki H, Shimotsuji S, Hori O. A Shot classification method of selecting effective key frames for video browsing. In Proc. ACM Multimedia 1995

3. Berchtold S, Keim D, Kriegel HP. The X-tree: an index structure for high-dimensional data. In Proc. $22^{\text {nd }}$ VLDB Conference, Mumbai, India, 1996

4. Correia N, Caetano A, Grilo A, Guimarães N. An environment for building reusable video information models. In ACM Symposium on Applied Computing SAC'98, San Francisco, USA, 1998

5. O'Connor B. Selecting key-frames of moving image documents: a digital environment for analysis and navigation. In Microcomputers for Information Management, 8(2), 1991

6. Faloutsos $\mathrm{C}$ et al. Efficient and effective querying by image content. In Journal of Intelligent Information Systems, 3, 1994

7. Flickner $\mathrm{M}$ et al. Query by image and video content: the QBIC system. In IEEE Computer, 28(9), 1995

8. Ioka M. A method of defining the similarity of images on the basis of color information. In Technical Report RT-0030, Tokyo Research Laboratory, Tokyo, Japan, 1989

9. Gong Y et al. An image database system with content capturing and fast image indexing abilities. In Proc. International Conference on Multimedia Computing and Systems, 1994

10. Gorkani M, Picard R. Texture orientation for sorting photos at a glance. In Proc. $112^{\text {th }}$ International Conference on Pattern Recognition, 1994 
11. Davis M. Media Streams: Representing video for retrieval and repurposing. PhD Thesis, Massachusetts Institute of Technology, Cambridge, Massachusetts, USA, 1995

12. Miyahara M, Yoshida Y. Mathematical transform of $(R, G, B)$ color data to Munsell $(H, V, C)$ color data. In SPIE Visual Communication and Image Processing, 1991

13. Minka T, Picard R: Interactive learning using a society of models”. In Proc. IEEE CVPR ‘96, 1996

14. Nagasaka A, Tanaka Y. Automatic video indexing and full-video search for object appearances. In Visual Database Systems II, Eds. Knuth E and Wegner L, Elsevier Science Publishers, 1992

15. Pentland A, Picard R, Scarloff S. Photobook: Tools for content-based manipulation of image databases. In IS\&T/SPIE Symposium on Electronic Imaging Science and Technology, San Jose, USA, 1994

16. Rowe L, Boreczky J, Eads C. Indexes for user access to large video databases. In IS\&T/SPIE Symposium on Storage and Retrieval for Image and Video Databases II, San Jose, USA, 1994

17. Rui Y, Huang T, Mehrotra S, Ortega M. A relevance feedback architecture in content-based multimedia information retrieval systems. In IEEE Workshop on Content-based Access of Image and Video Libraries, in conjunction with IEEE CVPR'97, 1997

18. Stricker M, Orengo M. Similarity of color images. In IS\&T/SPIE Symposium on Storage and Retrieval for Image and Video Databases III, San Jose, USA, 1995

19. Tamura H, Mori S, Yamawaki T. Texture features corresponding to visual perception. In IEEE Transactions on Systems, Man and Cybernetics, 8(6), 1978

20. White D, Jain R. Algorithms and strategies for similarity retrieval, Technical Report VCL-96-101, Visual Computing Laboratory, University of California, San Diego, 9500 Gilman Drive, Mail Code 0407, La Jolla, CA 92093-0407, 1996 (http://www-vision.ucsd.edu/papers/simret/)

21. Xiong W, Lee C, Ip M. Net comparison: A fast and effective method for classifying image sequences. In IS\&T/SPIE Symposium on Storage and Retrieval for Image and Video Databases III, San Jose, USA, 1995

22. Yeo B, Liu B. Rapid scene analysis on compressed video. In IEEE Transactions on Circuits and Systems for Video Technology, 1996

23. Yeung M, Yeo B, Liu B. Extracting story units from long programs for video browsing and navigation. In Proc. IEEE Conf. On Multimedia Computing and Systems, 1996

24. Zhang HJ et al. Automatic parsing and indexing of news video. In Multimedia Systems, 2, 1995

25. Zhang HJ et al. Video parsing using compressed data. In IS\&T/SPIE Symposium on Electronic Imaging Science and Technology: Image and Video Processing II, San Jose, USA, 1994

26. Zhang HJ, Kankanhalli A, Smoliar S. Automatic partitioning of full-motion video. In ACM/Springer Multimedia Systems, 1(1), 1993

27. Zhang HJ, Low C, Smoliar S, Wu J. Video parsing, retrieval and Browsing: an integrated and contentbased solution. In Proc. ACM Multimedia ‘95, San Francisco, USA, 1995 